\title{
ALGUNAS PALABRAS DESCONOCIDAS DE JORGE CÁCERES Y AIMÉ CÉSAIRE EN EL CONTEXTO INTERNACIONAL DEL SURREALISMO
}

\author{
Lucía Palenzuela \\ Universidad de La Laguna
}

\section{RESUMEN}

En su exilio americano, André Breton impulsa la creación de la revista $V V V$ (1942-1944) en Nueva York y mantiene el contacto con otras publicaciones americanas de la época que se expresan en lengua inglesa, francesa y también en español. El encuentro con las cartas del fondo André Breton conservadas en la Bibliothèque Littéraire Jacques Doucet de París revela que el contacto con el grupo chileno de Mandrágora y con el martiniqués Aimé Césaire fue continuado. Jorge Cáceres, Braulio Arenas y Enrique Gómez-Correa participan en $V V V$, de la misma manera que André Breton y Benjamin Péret publican en Leit Motiv. Estos autores están presentes y participan en este capítulo de la historia del surrealismo internacional. Asimismo, la relación con Aimé Césaire muestra los contactos intensos a lo largo del despliegue surrealista en América, ya en Tropiques o en $V V V$. Las cartas de Jorge Cáceres y de Aimé Césaire citadas parcialmente en este artículo, aportan nueva información acerca del tema.

Palabras clave: surrealismo, América, Tropiques, Leit Motiv, VVV, André Breton, Jorge Cáceres, Aimé Césaire.

\section{SOME UNKOWN WORDS FROM JORGE CÁCERES AND AIMÉ CÉSAIRE IN THE INTERNATIONAL CONTEXT OF SURREALISM}

\section{Abstract}

In his American exile, André Breton encourages the creation of the $V V V$ journal (1942-1944) in New York and gets in touch with some other American journals of the period expressing themselves in English, French and also in Spanish. The knowledge of the André Breton's letters preserved in Bibliothèque Littéraire Jacques Doucet in Paris proves that the contact with the Chilean group of Mandrágora and the Martinican Aimé Césaire was constant. Jorge Cáceres, Braulio Arenas and Enrique Gómez-Correa take part of $V V V$, just as André Breton and Benjamin Péret publish in Leit Motiv. These authors are present and participate in this chapter of the international Surrealism's history. The relationship with Aimé Césaire shows the intense contacts during the Surrealism's expansion. The letters from Jorge Cáceres and Aimé Césaire, partially reproduced in this article, provide new information about the topic. Keywords: Surrealism, America, Tropiques, Leit Motiv, VVV, André Breton, Jorge Cáceres, Aimé Césaire. 
En los años de publicación de $V V V(1942-1944)$, la revista editada en Nueva York, el surrealismo se había convertido ya en un importante movimiento de calado internacional. Las exposiciones internacionales surrealistas realizadas en Copenhague (1935), en Tenerife (1935), en La Louvière (1935), en Londres (1936), en Tokio (1937), en Ámsterdam (1938), en México D.F. (1940) y en Nueva York (1942) dan buena cuenta de ello (Sebbag 1994). En fechas anteriores a la llegada de los surrealistas a América se publican cuatro boletines internacionales, en Praga, en Santa Cruz de Tenerife, en Bruselas y en Londres. En todos estos acontecimientos participan los más destacados escritores y artistas que forman entonces parte del surrealismo. Desde diversas perspectivas, han tratado este proceso Juan Manuel Bonet en El surrealismo entre Viejo y Nuevo Mundo (Bonet 1988), Serge Fauchereau en su estudio publicado en La révolution surréaliste (Fauchereau 2002), Miguel Pérez Corrales en Caleidoscopio surrealista (Pérez Corrales 2011) o Eduardo Becerra en El surrealismo y sus derivas (Becerra 2013).

En América Latina la adhesión de artistas y escritores al surrealismo es constante desde mediados de los años 20. Algunos escritores latinoamericanos contactan con miembros del grupo de París, como Alejo Carpentier, aunque nunca llegó a formar parte del surrealismo. Diversos grupos se constituyen en el continente; en Argentina, con el decidido apoyo de Aldo Pellegrini; en Perú, bajo la influencia de César Moro; en Santiago de Chile, con los animadores de Mandrágora; en Fort-deFrance, con la colaboración de Aimé Césaire. Decisivos fueron también los viajes de los surrealistas europeos por los distintos países de Latinoamérica.

El exilio y la llegada de los surrealistas del grupo de París a América sigue una trayectoria particular que conviene recordar. El estallido de la Segunda Guerra Mundial y la ocupación de Francia en 1940 motiva la diáspora. Muchos se instalan inicialmente en Marsella. André Breton junto a Jacqueline y Aube llegan a la Villa Air Bel, sede del Comité americano de Rescate de intelectuales, dirigido por Varian Fry y Daniel Benedite. Coinciden ahí con Peggy Guggenheim y Max Ernst (Schuster 1989:70). Mantiene entonces contacto con amigos y personajes de uno y otro lado del Atlántico. Con César Moro y Wolfgang Paalen proyectan la Exposición surrealista de 1940, en México. Desde entonces los contactos se multiplican. Yves Tanguy, Kurt Séligmann están en Estados Unidos. Alfred Barr Jr., director del MoMA, trata de invitar a André Breton para dictar algunas conferencias en Nueva York.

En Marsella, Breton se encuentra con Óscar Domínguez, Bejamin Péret, André Masson, Marcel Duchamp, René Char, Victor Brauner, Wifredo Lam, etc. Es la época en la que realizan el juego de cartas «Jeu de Marseille», inspirado en el tarot, que será publicado con $V V V$. Es la época también en la que intenta publicar, sin éxito, Anthologie de l'humour noir y en la que acaba Pleine Marge. En 1941, publica en Marsella, en Éditions du Sagittaire, Fata Morgana, ilustrado por el cubano Wifredo Lam. Poco después de obtener un visado americano, André Breton embarca a bordo del Capitaine Paul Lemerle con destino a Martinica, donde conocerá y entablará amistad con el poeta de la Negritud, Aimé Césaire. En el barco que los lleva a la isla viajan, entre otros, Victor Serge, Wifredo Lam y Claude Lévi-Strauss. 
La coincidencia con Lévi-Strauss es muy singular. Breton y Lévi-Strauss no se conocen inicialmente. Así lo revela Lévi-Strauss en una entrevista concedida a Didier Éribon:

André Breton, j'ignorais qu'il fût à bord. C'est lors d'une escale au Maroc, où seuls les Français étaient autorisés à descendre à terre que, faisant la queue pour montrer mon passeport, je l'ai entendu, juste devant moi, dire son nom. [...] Je me suis présenté à lui et nous avons sympathisé (Lévi-Strauss 2009: 45).

Sobre este encuentro hablará Lévi-Strauss en diversos momentos. En Tristes Tropiques se refiere al inicio de «une durable amitié [qui] allait commencer par un échange de lettres qui se prolongea assez longtemps au cours de cet interminable voyage, et où nous discutions des rapports entre beauté esthétique et originalité absolue» (Lévi-Strauss 1955: 20). En Regarder, écouter, lire se recoge la nota sobre este encuentro y, también, la respuesta de André Breton (Lévi-Strauss 1994: 100-108).

Cuando el barco llega a Fort-de-France, Breton es considerado como un agitador y es internado en un campo de concentración durante algunos días. A su liberación, se produce el encuentro con Aimé Césaire, René Ménil y Suzanne Césaire y la recién fundada Tropiques. Se trata de un verdadero acontecimiento. Breton colabora a partir de entonces en la revista. En «Un grand poète noir» habla de Cahier d'un retour au pays natal, de Aimé Césaire, de su publicación en París en 1939 (Breton 1999: 400-408). Las circunstancias del encuentro con los animadores de Tropiques se muestran al principio del texto:

C'est dans ces conditions qu'il m'advint, au hasard de l'achat d'un ruban pour ma fille, de feuilleter une publication exposée dans la mercerie où ce ruban était offert. Sous une présentation des plus modestes, c'était le premier numéro, qui venait de paraître à Fort-de-France, d'une revue intitulée Tropiques [...]. La mercière martiniquaise, par une de ces chances accessoires qui accusent les heures fortunées, ne devait pas tarder à se faire connaître pour la sœur de René Ménil, avec Césaire le principal animateur de Tropiques. Et en effet, moins d'une heure plus tard, s'étant mise à ma recherche par les rues, elle m'indiquait de la part de son frère un rendez-vous.

Tras el encuentro, Breton se refiere a Aimé Césaire como «la cuve humaine portée à son point de plus grand bouillonnement». También dedica su atención a dos figuras importantes de Tropiques: Suzanne Césaire, a la que le dedica un texto poético, y René Ménil, al que vincula a «la grande culture en ce qu’elle a de moins ostentatoire, la mesure impeccable» (Breton 1999: 401).

Este primer encuentro con el mundo de Martinica en 1941 da como resultado un libro de referencia: Martinique charmeuse de serpents, donde vuelve a recoger los textos publicados en Tropiques. No se publicará de forma inmediata. Más tarde, en 1948, después del regreso de André Breton a Francia, aparece en Éditions du Sagittaire, en colaboración con André Masson, que no solo ilustra el libro, sino que escribe uno de los textos poéticos iniciales.

Después de su paso por el Caribe, André Breton llega a Nueva York a principios de julio de 1941 y se encuentra con Yves Tanguy, Kay Sage y Stanley William 
Hayter. En sus primeros meses en Nueva York la revista View se hace eco de la presencia del autor de Nadja y dedica su número doble 7-8 al movimiento surrealista. En la ciudad americana los exiliados surrealistas permanecen en contacto. Robert Motherwell recuerda que en los ańos 40 prácticamente todos los interesados por el arte en Nueva York se conocían. Coincidían en galerías de arte, en inauguraciones, en librerías o bien se encontraban por los alrededores de Greenwich Village, donde vivían. En palabras de Motherwell, el ambiente de Greenwich Village recordaba a la época parisina y a Saint-Germain-des-Prés. Ahí se instala André Breton.

A finales de 1941, Breton expresa su idea de realizar una revista surrealista en Nueva York y pide apoyo a los animadores del grupo. Durante el proceso de gestación de la publicación, el escritor francés participa en el programa radiofónico del periodista exiliado Pierre Lazareff «La Voix de l’Amérique parle aux Français». Junto a Georges Duithuit, Claude Lévi-Strauss y Robert Lebel, Breton lee textos de información y propaganda destinados a Francia y a los exiliados en América. Desde N.Y. Breton comienza a establecer contactos con los escritores latinoamericanos, en Chile, en México, en Martinica, en Cuba.

En marzo de 1942 aparece finalmente la primera entrega de la revista $V V V$. Se publica en Nueva York entre junio de 1942 y febrero de 1944. La revista se encuentra en lengua inglesa y francesa. A diferencia de otras publicaciones de la época, como la mexicana Dyn, que traduce los textos en ambas lenguas, los textos pueden hallarse en la lengua original, sin presentarse en una edición bilingüe. Los creadores anglófonos y francófonos suelen escribir en su lengua materna. Sin embargo, no debe extrañarnos si encontramos artículos de autores franceses en inglés. Este es el caso, por ejemplo, de Claude Lévi-Strauss. Hay algún artículo en lengua española, utilizada por los escritores latinoamericanos.

A partir de la llegada de André Breton a Nueva York, la relación con Aimé Césaire continúa a través de un dilatado epistolario. Los temas que abordan tienen que ver con la situación política, con las colaboraciones en las revistas y con los intereses surrealistas.

La preocupación de Césaire por la situación cultural y política de las Antillas es notable y evidente en las cartas enviadas a Breton. El escritor martiniqués explica ampliamente cómo se ha desarrollado la vida en Martinica bajo el régimen de Vichy. En agosto de 1943 escribe:

Le régime que nous avons subi ici pendant trois ans explique la rareté et l'insignifiance de nos lettres. Le programme vous est connu. Il a été appliqué intégralement: surveillance policière, délation, racisme; du point de vue intellectuel, obscurantisme; censure pour la presse; censure pour Tropiques, 20 fois inutile, finalement interdit. D'une manière générale, isolement savant, vase-clos, nuit noire [...] Dans l'ensemble, quelque chose d'immonde et de gluante (Carta de Aimé Césaire 3/08/1943).

Poco después, en septiembre de 1943, Aimé Césaire afirma que, en efecto, «décidément les temps ont bien changé» (Aimé Césaire 5/09/1943). La caída del gobierno de Vichy en las Antillas hace pensar que los tiempos han cambiado. Sin embargo, el gobierno local es aún inestable y la situación continúa siendo difícil: 
Le gouvernement ne fait montre ni de l'audace ni de l'esprit d'initiative que requiert la situation. L'épuration impatiemment attendue est à peine entamée. Les abus économiques du régime défunt continuent. Du point de vue social, conservatisme ridicule: on flirte avec les grands féodaux du sucre et du rhum, même quand officiellement on affecte de flétrir le capitalisme. L'ouvrier agricole continue à toucher 15 francs par jour. Et cela on ne peut même pas le crier dans les journaux. Censure. Les salauds de Vichy restent en place, plus arrogants que jamais. Le gouvernement a changé -et me semble brave homme- les bureaux n'ont pas changé (Aimé Césaire $16 / 11 / 1943)$.

En 1945, Aimé Césaire no percibe aún grandes transformaciones y comenta: «C'est un fait: nous sommes à un confluent boueux. Il faut être doucement cinglé pour croire à une quelconque régénération» (Aimé Césaire 2/04/1945). Considera, asimismo, que la situación de la sociedad occidental es insostenible: «C'est terrible, un zombi. Tout cela pèse plus lourdement que jamais sur le destin de nos Antilles».

Bajo estas circunstancias tiene lugar el encuentro con el surrealismo. La influencia surrealista en la revista publicada por Aimé Césaire en Fort-de-France, Tropiques (1941-1945), es muy evidente a partir del tercer número, esto es, tras el encuentro con André Breton. Las alusiones al movimiento y a sus principios son numerosas y se manifiestan de forma diversa. Las reflexiones sobre el surrealismo revelan el punto de vista del poeta martiniqués en esos tiempos de guerra:

Il est exaltant de penser que maintenant comme au lendemain de la lère guerre mondiale, c'est le surréalisme qui prend acte, le premier de la démission du monde; qu'il garde l'initiative d'une révision des valeurs; tâche de déterminer le sens d'une dynamique nouvelle, et de sauver ce qui peut être encore sauvé de la pureté et de l’innocence (Aimé Césaire 3/08/1943).

Aimé Césaire piensa que el surrealismo abre una nueva vía en la poesía. De esta manera, cuando se anuncia la muerte del surrealismo, cuando se habla de la situación del surrealismo en el periodo de entreguerras, Césaire responde: «Non, le surréalisme n'est pas mort; et il s'affirme de plus en plus notre seul espoir en ce moment de plus grande "fragilité" du monde; en ce moment où singulièrement l'orientation de la poésie me semble infiniment dangereuse» (Aimé Césaire 22/09/1943).

La relación de Césaire y Breton en esta época se basa no solo en la admiración mutua sino también en el intercambio. Breton publica en Tropiques y Césaire en $V V V$. Los dos intercambian obra, los dos se citan y dedican poemas. Después de la recepción del texto «Un grand poète noir», Aimé Césaire escribe:

Mieux qu'une présentation, votre préface est pour moi une révélation. Je veux dire que par elle, vous m'avez aidé à voir clair en moi-même. Alors que certains croyaient devoir signaler dans mon attitude une contradiction fondamentale (poète nègre-poète surréaliste) -et l'on me dit tous les jours narquoisement pourquoi un poète nègre s'embrasserait-il du surréalisme?- vous m'apportez la solution. Cette fausse antinomie, je la sentais fausse, bien sûr; je la sentais fausse, et j'ai tenté bien des fois -hélas! maladroitement- d'en exprimer l'inanité. Et ce que je sentais, voici que vous le dites lumineusement. De manière, croyez-le, bouleversante pour moi (Aimé Césaire 4/04/1944). 
Asimismo, el poeta de Fort-de-France reconoce la influencia que el surrealismo ha ejercido sobre él y su obra: "C’est le surréalisme qui, lors même que je ne songeais pas à m'en revendiquer, m'a vraiment imprégné de cette idée qu'il pouvait, qu'il devait exister une poésie dénouée. Alors, et alors seulement, physiquement, $j$ 'ai pu parler, écrire». Aimé Césaire afirma igualmente que su escritura se encuentra próxima de los principios surrealistas:

J'avais choisi le silence, quand une fréquentation plus assidue du surréalisme, puis votre rencontre décisive intervinrent. Se laisser parler. Se laisser envahir par ses rêves. Se laisser dominer par ses images. Il n'était plus question de «thèse», ni de "thème». Il s'agissait tout simplement d'oser la vie, toute la vie. J'étais libre de nouveau. Totalement. Définitivement libre et renouvelé. Si bien que maintenant, pour moi, surréalisme, liberté, poésie sont trois termes qui n'en font qu'un. Et ce n'est pas pour moi une vérité seulement lue dans le Manifeste du Surréalisme. C'est une idée que j'ai vécue, que j'ai conquise. C'est le bilan de mon expérience que je vous livre (Aimé Césaire 4/04/1944).

La relación epistolar de Césaire y Breton está marcada por silencios y algunos desacuerdos. Los silencios pueden ser explicados quizá por el estado de las comunicaciones bajo el régimen de Vichy. Aimé Césaire tiene problemas para recibir las cartas de Breton y le pregunta en varias ocasiones: «Qu'y a-t-il? Toujours ce grand silence de vous" (Aimé Césaire 3/08/1944). Los desacuerdos tienen que ver probablemente con aspectos políticos. Desde 1945 a 1956, momento en el que envía una conocida Lettre à Maurice Thorez para romper con el Partido Comunista, Césaire se integra en el PC. Este vínculo con el Partido es probablemente el motivo del distanciamiento con Breton. En 1948, Césaire escribe al poeta francés:

Mon cher André, un dissentiment existe entre nous sur un point très précis: je crois en conscience et je vous l'ai déjà dit, que je n'ai moralement pas le droit d'adopter la position politique qui est la vôtre. A l'heure actuelle, il se livre un grand combat, et [...] je pense n'avoir pas le droit de m'en abstraire. Cela dit, j'ai toujours voulu que ce désaccord restât limité et purgé de toute pensée mesquine (Aimé Césaire 6/10/1948).

André Breton había roto los lazos con el Partido Comunista. En 1935, en el Congreso internacional de escritores proletarios, Breton había manifestado la posición de los surrealistas. Estos no someterían la libertad a las directrices del Partido Comunista Francés y de la URSS.

En efecto, Breton y Césaire divergen en este punto preciso. El primero había abandonado al Partido Comunista y el otro acaba de ingresar. Breton no considera admisibles las tesis del PC. Aimé Césaire las suscribe. Las relaciones no volverán a ser iguales.

Entre tanto, hubo colaboración en las revistas Tropiques y $V V V$. El surrealismo se encuentra también con otros núcleos de creadores. André Breton toma contacto con los chilenos y especialmente, como se muestra en las cartas que hemos podido leer en la Bibliothèque Littéraire Jacques Doucet, con Jorge Cáceres. 
Jorge Cáceres, Braulio Arenas, Enrique Gómez-Correa y Teófilo Cid son los animadores de la revista Mandrágora, que, como es conocido, se publica en Santiago de Chile entre 1938 y 1943. Se trata de una publicación que, desde el primer momento, antes incluso de entrar en contacto con los surrealistas, se encuentra cercana a las inquietudes del movimiento. De la misma manera que en $V V V$, en Mandrágora se reproducen textos de Alfred Jarry y se habla de Une saison en enfer, de Arthur Rimbaud; y se realizan, además, reseñas de obras de Paul Éluard y André Breton, concretamente de Cours naturel y L'Amour-fou. La revista que sucede en Chile a Mandrágora es Leit Motiv, publicada entre 1942 y 1943. En el número doble de diciembre de 1943, podemos encontrar un mensaje publicitario de $V V V$ en las últimas páginas: se hace referencia al número 2-3, al editor David Hare, a los miembros del comité de edición André Breton, Max Ernst, Marcel Duchamp: a colaboradores como Leonora Carrington, Aimé Césaire, Wifredo Lam, Benjamin Péret y a los propios chilenos del grupo Mandrágora, que contribuyen de igual forma en la revista neoyorkina.

El contacto con André Breton se establece a través del poeta Benjamin Péret, que colabora ya con los escritores del grupo. Jorge Cáceres envía una carta a André Breton desde Santiago el 27 de agosto de 1942. Esta carta, como las de Césaire, se halla en la Bibliothèque Littéraire Jacques Doucet de París. Pertenece al fondo André Breton. Descubrimos que el animador de Mandrágora ya había intentado establecer contacto a través del envío de diversas publicaciones:

Yo espero que Ud. haya recibido los numerosos panfletos que yo le he enviado a diversas direcciones, entre ellas «Galerie Matisse» y la que me ha proporcionado Péret, con quien mantengo correspondencia, y quien nos acaba de enviar un admirable poema para nuestra revista, próxima a aparecer (Cáceres 27/08/1942).

Se trata del poema «Premiers résultats», que se publica en el primer número de Leit Motiv acompañado del dibujo El palacio de Peau d'Âne, de Jorge Cáceres. Percibimos tanto en el epistolario como en Leit Motiv el diálogo entre surrealistas. $\mathrm{El}$ autor chileno conoce $V V V$ a través de personas que poco tienen que ver con el surrealismo. Desde la primera carta a la que hemos tenido acceso, advertimos que intenta recibir la revista:

\section{Cher Breton:}

Es en el momento en que personas que no pertenecen al grupo surrealista chileno, acaban de recibir vuestra revista " $V V V$ », que yo me dirijo a $\mathrm{Ud}$.

Acabo, también, de leerla, y me es preciso confesaros que ella llena absolutamente el molde de mis esperanzas. [...]

Vuestra revista me interesa de sobremanera, por lo cual os ruego vivamente enviarme un ejemplar a la siguiente dirección:

Jorge Cáceres

Casilla 9590

Correo Central

Santiago de Chile (Cáceres 27/08/1942) 
Se conservan en el Fondo André Breton un total de cinco cartas, remitidas a su dirección de Nueva York. No son numerosas si se tiene en cuenta el periodo en el que son escritas, entre 1942 y 1944. En la segunda carta, redactada el 7 de noviembre de 1942, Jorge Cáceres y el grupo Mandrágora contestan a una petición de colaboraciones para $V V V$ de André Breton: "Como Ud. me lo pide, le envío aquí una "Lettre du Chili", en la cual mi amigo Braulio Arenas rinde cuenta a Ud. de nuestra actividad desde el año 1938. Acompañan a esta, numerosos documentos fotográficos que yo he escogido rápidamente, y algunos textos de Arenas, GómezCorrea y míos» (Cáceres 7/11/1942).

En efecto, si confrontamos estos documentos, descubrimos que «Letter from Chile» es publicada en el número de 1943. Se toma la fecha de la carta de Cáceres a Breton, «Santiago, 7 de noviembre 1942», y se reproduce, como indica Jorge Cáceres a André Breton, la relación de actividades surrealistas desarrolladas por los autores chilenos desde 1938 a septiembre de 1942. Las palabras escritas por Braulio Arenas se centran en la constitución del grupo surrealista y en su trabajo en medio de «la incomprensión y hostilidad más grandes, manifestadas por el medio en que estas actividades se desarrollaban. Es por esta razón que nosotros consideramos doblemente precioso el estímulo vuestro hacia nuestra labor» (Cáceres 7/11/1942). Braulio Arenas muestra aquí el alejamiento de los animadores de Mandrágora de las posturas de Pablo Neruda y de la influencia del comunismo internacional. En cierto modo, los surrealistas chilenos actúan de manera similar a André Breton y los surrealistas franceses a principios de 1930 cuando critican a Louis Aragon, el estalinismo y el realismo socialista. Escribe Arenas en «Letter from Chile»:

1940, julio. Arenas, Gómez y Onfray interrumpen un acto de homenaje a Pablo Neruda (Universidad de Chile), pidiendo que Neruda dé cuenta del resultado de las colectas que organizaba en favor de los niños españoles, en su calidad de Presidente de la Alianza de Intelectuales de Chile. Este homenaje se rendía a Neruda con motivo de su designación como Cónsul General de Chile en México. Braulio Arenas rompió la conferencia que Neruda leía, con lo que se suspendió el acto (Arenas 1943a: 124).

Bajo el título «Letter from Chile» se publican entonces en $V V V$ las palabras de Braulio Arenas y tres poemas: «La mejor parte», de Jorge Cáceres (1943b: 125), «El prestigio del cuerpo humano», de Enrique Gómez-Correa (1943: 126), los dos publicados en lengua española, y en inglés y dedicado a Benjamin Péret, «The Mystery of The Yellow Room", de Braulio Arenas (1943b: 126). No se encuentran, sin embargo, las ilustraciones, las fotografías y los collages que Jorge Cáceres anunciaba en su carta.

En la carta del 24 de diciembre de 1942, Cáceres manifiesta su inquietud con respecto a la ausencia de respuesta de Breton. Teme que toda la documentación se haya perdido y que el francés no la haya recibido a causa de las «intrincadas e informales redes de los correos internacionales, o bien haber sido rechazado por la censura sospechosa de su insólito contenido». En esta misiva Cáceres ańade que «nuestra revista» está a punto de salir, que se podrán leer textos de Péret y tam- 
bién «SUS PROLEgómeNOS A UN TERCER MANIFIESTO [que] han sido magníficamente traducidos al español por Arenas; él ha tomado en cuenta para dicha traducción los textos en inglés y en francés publicados en $V V V$ ». Sin duda, la revista que está a punto de salir es Leit Motiv. Editada en diciembre de 1942, en efecto, en el número 1 de Leit Motiv se publica el poema citado de Péret y «Prolegómenos a un tercer manifiesto o no".

En la misma carta, Cáceres pide textos inéditos para el segundo número de la revista y pide también las direcciones de amigos surrealistas con el fin de solicitar colaboración. De la misma manera, indica que enviará Leit Motiv a Paalen, «a Benjamin, a Moro, a Caillois, a Westphalen y otros». El surrealismo en América tiene ya una dimensión internacional.

En 1943, en una interesante carta donde intercala dibujos con el texto, Cáceres habla de la recepción del número 2-3 de $V V V$. Es el número en el que colaboran los chilenos («Le agradezco nuevamente el habernos publicado», dice). Sus elogios se dirigen ahora a dos colaboradores de $V V V$, el también chileno Roberto Matta y a Charles Duits: «Los dibujos de Matta me parecen geniales, como también la presencia de Charles Duits». Anuncia el número 2 de Leit Motiv y la contribución de Péret con su «Dernier malheur, dernière chance», que había sido publicado en el número 2-3 de $V V V$ y que será reproducido de nuevo en la revista chilena. La petición que había realizado en la carta precedente obtiene una respuesta positiva. En el mismo número de Leit Motiv, encontramos «Primeros transparentes», de Breton, un texto dedicado a Charles Duits, traducido al español por Enrique Rosenblatt.

En la carta de Cáceres a Breton, se anuncia asimismo la recepción de un poema de Aimé Césaire, el director de Tropiques en Martinica y colaborador de $V V V$. En efecto, encontramos "Colombes bruissement du sang», del martiniqués, en el número de diciembre de 1943 de Leit Motiv. Se trata de una colaboración especial para la publicación chilena, pues este texto no se publica ni en Tropiques ni en $V V V$. Suponemos que André Breton pone en contacto a Jorge Cáceres y a Aimé Césaire, con el que mantenía estrecho contacto en esta época, como demuestran las cartas anteriormente citadas. Jorge Cáceres colabora también en la martiniquesa Tropiques. En el número de Tropiques de octubre de 1943 se publica en español «Una alondra polar me ha saludado al pasar», en traducción francesa de René Durand (Cáceres 1943a: 19-22).

La última carta, que hemos encontrado en el Fondo de la Bibliothèque Jacques Doucet enviada por Jorge Cáceres a André Breton a su dirección de Nueva York, data de 1944. Cáceres afirma que ha recibido una carta de Péret «pero él no me habla absolutamente nada acerca de Usted y de sus actividades. Yo estoy francamente preocupado por su silencio». Además de la preocupación personal por Breton, señala que ha recibido el catálogo de Enrico Donati «que contiene su prólogo». El autor chileno da también otros datos de interés: Braulio Arenas parte para Buenos Aires y Cáceres será a partir de ahora el responsable de la revista. El autor tiene la intención de dedicar un número a Roberto Matta, «el primer homenaje a ese gran pintor en su propia patria». Solicita ahora colaboraciones con ese propósito: «Le ruego que le pida a Matta, a Ernst, a Duits, etc. algo para nosotros, pero esto debe ser rápido; a vuelta de correo» (Cáceres 2/04/1944). 
La realidad es que Leit Motiv se publica por última vez en 1943 y que el número homenaje a Matta no se editaría. En todo caso, las cartas que Jorge Cáceres remite desde Santiago a Nueva York revelan la conexión que existió entre los grupos próximos al movimiento surrealista. La colaboración del martiniqués Aimé Césaire en la revista chilena y de Cáceres en Tropiques muestra también la dimensión internacional del surrealismo que se expresa ya en diversas lenguas. Sucede lo mismo con la publicación de textos de Breton en Leit Motiv y la «Letter from Chile» en VVV.

Las cartas, que se conservan en los fondos de la Bibiothèque Littéraire Jacques Doucet de París, permiten conocer mejor la expansión del movimiento surrealista internacional. Hemos querido mostrar aquí, a través de la consulta de los fondos de André Breton y de la consulta de las revistas $V V V$ y aquellas otras con las que mantiene un estrecho diálogo (Leit Motiv, Tropiques), cómo los vínculos entre los creadores surrealistas se intensifican en el comienzo de los ańos 40 . Hemos pretendido mostrar, a través de las cartas inéditas conservadas en París, este importante capítulo del desarrollo del movimiento surrealista en América.

ReCibido: diciembre de 2018; ACEPTADo: abril de 2019. 


\section{BIBLIOGRAFÍA}

Arenas, B. (1943a): «Letter from Chile», $V V V, 2-3,124$.

Arenas, B. (1943b): «The Mystery of the Yellow Room», VVV, 2-3, 126.

BeCERRA, E. (coord.) (2013): El surrealismo y sus derivas: visiones, declives y retornos, Madrid: Abada Editores.

Bonet, J.M. et al. (1989): El surrealismo entre Viejo y Nuevo Mundo, Las Palmas de Gran Canaria: Centro Atlántico de Arte Moderno.

Breton, A. (1942): «Prolegómenos a un tercer manifiesto o no», Leit Motiv, 1, 15-23.

Breton, A. (1999): Euvres Complètes, III, édition de Marguerite Bonnet, Paris: Gallimard.

CÁceres, J. Carta inédita a André Breton. Fondo André Breton. Bibliothèque Littéraire Jacques Doucet, 27 de agosto de 1942.

Cáceres, J. Carta inédita a André Breton. Fondo André Breton. Bibliothèque Littéraire Jacques Doucet, 7 de noviembre de 1942.

CÁceres, J. (1943a): «Una alondra polar me ha saludado al pasar», Tropiques, 8-9, 19-22.

CÁceres, J. (1943b): «La mejor parte», VVV, 2-3, 126.

CÉSAIre, A. (1943): «Colombes bruissement du sang», Leit Motiv, 2-3, 9.

Césaire, A. Carta inédita a André Breton. Fondo André Breton. Bibliothèque Littéraire Jacques Doucet, 3 de agosto de 1943.

Césaire, A. Carta inédita a André Breton. Fondo André Breton. Bibliothèque Littéraire Jacques Doucet, 5 de septiembre de 1943.

CÉsaire, A. Carta inédita a André Breton. Fondo André Breton. Bibliothèque Littéraire Jacques Doucet, 22 de septiembre de 1943.

CÉsaire, A. Carta inédita a André Breton. Fondo André Breton. Bibliothèque Littéraire Jacques Doucet, 16 de noviembre de 1943.

CÉsaire, A. Carta inédita a André Breton. Fondo André Breton. Bibliothèque Littéraire Jacques Doucet, 4 de abril de 1944.

CÉsaire, A. Carta inédita a André Breton. Fondo André Breton. Bibliothèque Littéraire Jacques Doucet, 2 de abril de 1945.

CÉsaire, A. Carta inédita a André Breton. Fondo André Breton. Bibliothèque Littéraire Jacques Doucet, 6 de octubre de 1948.

ChÉNieux-Gendron, J. (1994): Le surréalisme autour du monde (1929-1947), Paris: CNRS Éditions, 1994.

Fauchereau, S. (2002): «Quelques semaines au printemps de 1935: l'International surréaliste», en La revolution surrealiste, Paris: Centre Pompidou, 403-411.

Fauchereau, S. (2004): Mexique-Europe. Allers-Retours 1910-1960, Paris: Éditions Cercle d'art.

Gómez-Correa, E. (1943): «El prestigio del cuerpo humano», $V V V, 2-3,126$.

Lévi-Strauss, C. (1955): Tristes Tropiques, Paris: Plon.

Lévi-Strauss, C. (1994): Mirar, escuchar, leer, Madrid: Ediciones Siruela.

LÉvi-Strauss, C., y Didier Éribon (2009): De près et de loin, Paris: Odile Jacob. 
Péret, B. (1942): «Premiers résultats», Leit Motiv, 1, 11.

PÉret, B. (1943): «Dernier malheur, dernière chance», Leit Motiv, 2-3, 17-22.

Pérez Corrales, M. (2011): Caleidoscopio surrealista. Una visión del surrealismo internacional (19192011), Santa Cruz de Tenerife: La Página.

Sebbag, G. (1994): Le surréalisme, Paris: Nathan.

Schuster, J. (1989): «La diáspora surrealista durante la Segunda Guerra Mundial», El surrealismo entre Viejo y Nuevo Mundo, Las Palmas de Gran Canaria: Centro Atlántico de Arte Moderno, 69-80.

SChWARTZ, J. (1991): Las vanguardias latinoamericanas, Madrid: Cátedra. 